\title{
Preventing structural aging with synthetic tooth enamel
}

\author{
Li-Bo Mao, Huai-Ling Gao and Shu-Hong Yu*
}

Teeth are typically non-regenerative and permanent skeletal structures and their resilience to aging is one of the top requirements for the survival of the species. Recent investigations on mechanical properties of laboratory-made teeth enamel analogs show that they have a unique combination of stiffness, $E$, and vibration damping, $\tan \delta[1]$; this combination provides the load bearing organs the ability to resist slowly accumulating damage due to omnipresent oscillatory stresses. However, stiffness and vibrational damping are known to be the properties contrarian to each other, i.e., improvement of one results in deterioration of the other. Furthermore, it is believed that it is impossible for materials to possess both of these properties beyond the limit of $E \cdot \tan \delta=0.6$ [2]. However, enamel and dentine in teeth show $E \cdot \tan \delta$ exceed such limit [3]. The enamel layer of teeth has unique architecture based on stiff inorganic columns with high inorganic volume content of 85 vol.\% [4]. Kotov and coworkers [1] at the University of Michigan found that the columnar orientation of enamel is preserved throughout the ages and among different living creatures. Columnar organization is also present in exoskeletons of long-living sea animals perhaps for the same purpose of preserving of their protective functions for extended period of time [5].

This work is a pioneering study on the new family of biomimetic composites. To understand better the breakthroughs made here, it is useful to mention that horizontally laminated structures found in a variety of organisms are serving as drivers of the technology in many areas. One well known example of biological composites that spurr the development of ultrastrong materials with the combinations of optical, electrical, and thermal properties, is nacre of abalone shell also known as mother-of-pearl. Nacre is composed of 300-900 nm thick $\mathrm{CaCO}_{3}$ platelets sandwiched with 10-30 nm thick organic layers. Being started as a fundamental academic study to understand better the origin of its toughness and iridescence, nacre replicas are now used not only in protective materials but also in electronics, energy storage, sensors, etc. In addition to high mechanical properties, the artificial nacre-like materials have also been used for aesthetic purposes, for instance in construction of iridescent walls of Guggenheim Museum in Bilbao. The production of nacre-like artificial material with exceptional combination of properties is now utterly scalable as exemplified by Mao et al. [6], utilizing a chitosan matrix for matrix-directed mineralization of $\mathrm{CaCO}_{3}$ aragonite microcrystals to mimic well-ordered laminated structures of nacre. Also other synthetic approaches have been developed to realize such planar structural motif in artificial nanocomposites $[7,8,9]$. However, studies on the artificial nanocomposites with columnar structures have been barely performed so far, although we can find such motif in a wide range of biomaterials.

Inspired by the vertical orientation as structural motif, Kotov and coworkers [1] synthesized artificial enamel biocomposites. They mimicked the vertical orientation of inorganic columns in enamel with vertically oriented zinc oxide $(\mathrm{ZnO})$ nanowires grown onto the substrates (Fig. 1). The protein-based organic matrix of biocomposites was replicated by layers of two polyelectrolytes, polyallylamine and polyacrylic acid infiltrated between $\mathrm{ZnO}$ nanowires using layer-by-layer (LBL) assembly method. After repetition of the growth of inorganic nanowires and infiltration process with organic materials, they succeeded to build $\mathrm{ZnO} / \mathrm{LBL}$ multilayered nanocomposites with inorganic volume content of 67 vol.\% and investigated static and

Division of Nanomaterials \& Chemistry, Hefei National Laboratory for Physical Sciences at the Microscale, Department of Chemistry, University of Science and Technology of China, Hefei 230026, China

*Email: shyu@ustc.edu.cn 

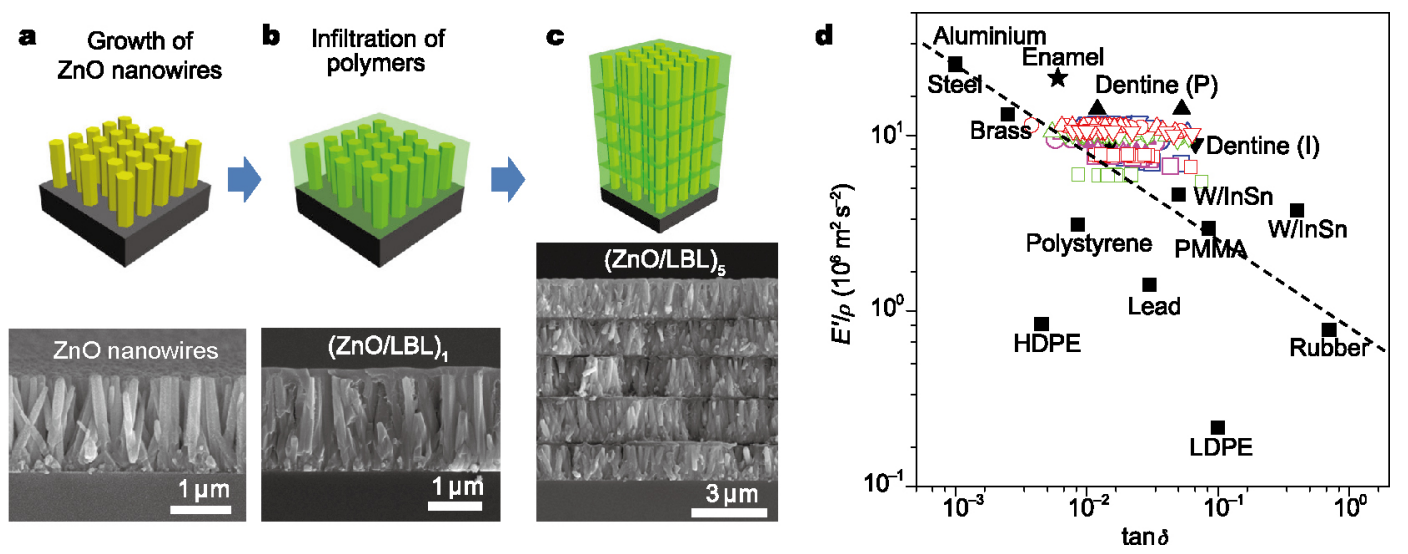

Figure $1(\mathrm{a}-\mathrm{c})$ Schematics of the synthesis process of artificial tooth enamel composites. Cross-sectional scanning electron microscopy images are shown at bottom row. (d) Damping coefficient $(\tan \delta) v s$. storage modulus divided by density $\left(E^{\prime} / \rho\right)$ plots. Dotted line indicates weight-adjusted viscoelastic figure of merit, VFOM, limit for conventional materials. Reprinted by permission from Ref. [1]. Copyright 2017, Nature Publishing Group.

dynamic mechanical properties of the $\mathrm{ZnO} / \mathrm{LBL}$ nanocomposite films. For static measurements, a modulus of $39.8 \mathrm{GPa}$ and a hardness of $1.65 \mathrm{GPa}$ for the $\mathrm{ZnO} / \mathrm{LBL}$ nanocomposites were obtained, which were comparable to the modulus of $62-108 \mathrm{GPa}$ and hardness of 1.1-4.9 GPa for tooth enamel (85 vol.\% of inorganic content) [10]. Oscillation damping coefficient, $\tan \delta$ is as high as 0.067 , which was 10-100 times higher than that of the conventional materials with similar moduli. Additionally, weight adjusted viscoelastic figure of merit (VFOM) exceeded the limit $\left(E^{\prime} / \rho\right) \times(\tan \delta)^{0.5}=0.8$, comparable to the values of dentin layer of tooth and outstripping the perceived boundary of conventional materials.

The method reported by Kotov et al. for making enamellike columnar nanostructures is of particular advantage in making large-area materials, which is essential for putting such biomimetic composites into practical use for a wide range of technological needs from vibration-resilient computer chips, safe batteries, and long-term biomedical implants to aviation and automotive industry. Given the scalability of the biomimetic materials [6,7], one can envision that the columnar architecture enamel can also inspire advances in the vibration isolation of the large scale structures including 3D-printed buildings.

Received 16 May 2017; accepted 17 June 2017; published online 4 July 2017

1 Yeom B, Sain T, Lacevic N, et al. Abiotic tooth enamel. Nature, 2017, 543: 95-98

2 Wang YC, Ludwigson M, Lakes RS. Deformation of extreme viscoelastic metals and composites. Mater Sci Eng-A, 2004, 370: 41-49

3 He LH, Swain MV. Understanding the mechanical behaviour of human enamel from its structural and compositional characteristics. J Mech Behav Biomedical Mater, 2008, 1: 18-29

4 Lussi A (eds.). Dental Erosion: From Diagnosis to Therapy. Basel: Karger, 2006

5 Weiner S, Addadi L. Design strategies in mineralized biological materials. J Mater Chem, 1997, 7: 689-702

6 Mao LB, Gao HL, Yao HB, et al. Synthetic nacre by predesigned matrix-directed mineralization. Science, 2016, 354: 107-110

7 Yan YX, Yao HB, Mao LB, et al. Micrometer-thick graphene oxidelayered double hydroxide nacre-inspired coatings and their properties. Small, 2016, 12: 745-755

8 Podsiadlo P, Kaushik AK, Arruda EM, et al. Ultrastrong and stiff layered polymer nanocomposites. Science, 2007, 318: 80-83

9 Finnemore A, Cunha P, Shean T, et al. Biomimetic layer-by-layer assembly of artificial nacre. Nat Commun, 2012, 3: 966

10 Angker L, Swain MV. Nanoindentation: application to dental hard tissue investigations. J Mater Res, 2006, 21: 1893-1905 